\title{
Proposed RF Breakdown Studies at the AWA
}

\author{
S. Antipov ${ }^{1,2}$, M. Conde ${ }^{1}$, V. Dolgashev ${ }^{3}$, W. Gai ${ }^{1}$, J. G. Power ${ }^{1}$, \\ L. Spentzouris ${ }^{1,2}$ and Z. Yusof ${ }^{1}$ \\ ${ }^{1}$ Argonne National Laboratory, Argonne IL 60439, USA \\ ${ }^{2}$ Illinois Institute of Technology, Chicago IL 60616, USA \\ ${ }^{3}$ Stanford Linear Accelerator Center, Menlo Park CA 94025, USA
}

\begin{abstract}
A study of breakdown mechanism has been initiated at the Argonne Wakefield Accelerator (AWA). Breakdown may include several factors such as local field enhancement, explosive electron emission, Ohmic heating, tensile stress produced by electric field, and others. The AWA is building a dedicated facility to test various models for breakdown mechanisms and to determine the roles of different factors in the breakdown. We plan to trigger breakdown events with a high-powered laser at various wavelengths (IR to UV) to determine the role of explosive electron emission in the breakdown process. Another experimental idea follows from the recent work on a Schottky-enabled photoemission in an RF photoinjector [1] that allows us to determine in situ the field enhancement factor on a cathode surface. Monitoring the field enhancement factor before and after the breakdown can shed some light on a number of observations such as the crater formation process.
\end{abstract}

Keywords: RF instrumentation, electron beams

PACS: 41.20.-q, 41.75.Fr

\section{DARK CURRENT}

In high gradient structures, in addition to the electron beam, dark current is also present. The physics of the formation of this dark current may vary: field emission, secondary emission, photoemission, etc. Typically, the characteristics of this dark current are quite reproducible from pulse to pulse. Images of the emitters do not change unless a breakdown happens. In the event of a breakdown, some emitters disappear and some are created. Several theories have been proposed to understand this process $[2,3]$.

Dark current has exponential dependence with surface electric fields. Experimental data are usually analyzed with the Fowler-Nordheim model [4] with two adjusted parameters: emitter area and field enhancement factor $\beta$. If this $\beta$ is multiplied by the maximum surface field reached in the structure $\left(\beta \cdot E_{\max }\right)$, the result is approximately 1 volt per angstrom $(10 \mathrm{GV} / \mathrm{m})$. This value appears to be a common level in many highgradient structures when breakdown is initiated. Relation between dark currents and breakdown is not well-understood. It is not known whether $\beta \cdot E_{\max }$ value is related to the trigger or the result of RF breakdown.

Contributed to 12th Advanced Accelerator Concepts Workshop (AAC 2006),

07/10/2006--7/15/2006, Lake Geneva, Wisconsin

Work supported in part by US Department of Energy contract DE-AC02-76SF00515 


\section{PROPOSED STUDIES}

Following the Fowler-Nordheim model, this experimental $\beta$ gives some average information about the field emitters. However, the number and distribution of the emitters are not known. Large experimental values of the $\beta$ currently do not have a pure geometric explanation. The "hairs" on surface of conductors, which would provide large geometrical values of $\beta$, are not observed from the experiment especially for high vacuum multi-megawatt RF structures.

We plan to conduct experiments using a high gradient RF gun with removable cathode to observe dark currents and breakdowns in a controlled environment. We will try to identify the emitters from the dark current data and compare their location with the SEM image of the cathode. This study is devoted to understanding the physical nature of Fowler-Nordheim $\beta$ and its dependence on the cathode material and surface preparation. This information should provide clues for understanding the relationship between the dark current and the breakdown.

\section{EXPERIMENTAL SETUP}

We are building a dedicated beamline with a high gradient $1 / 2$ cell $1.3 \mathrm{GHz}$ gun for study of microscopic properties of dark currents and breakdowns (Fig. 1). The dark current measurements for this gun were reported in Ref. [5].

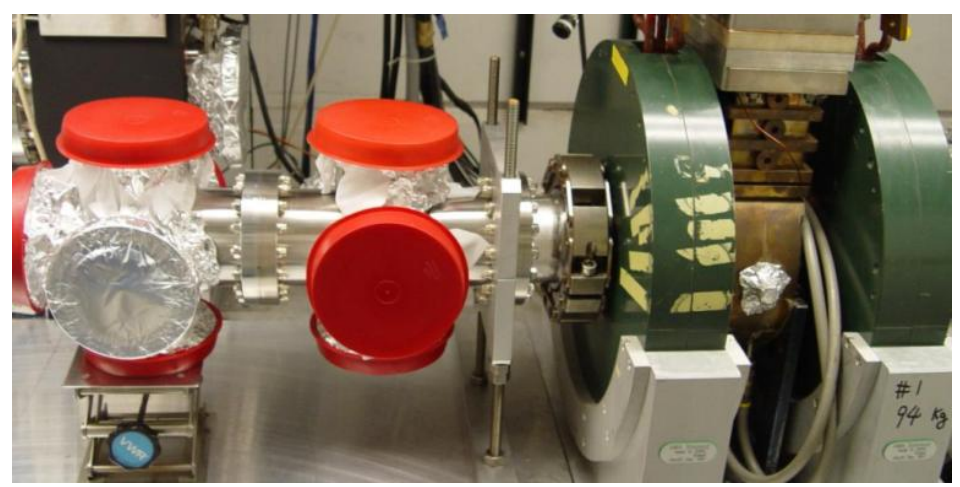

FIGURE 1. $1 / 2$ cell, $1.3 \mathrm{GHz}$ electron injector with a diagnostics chamber. 


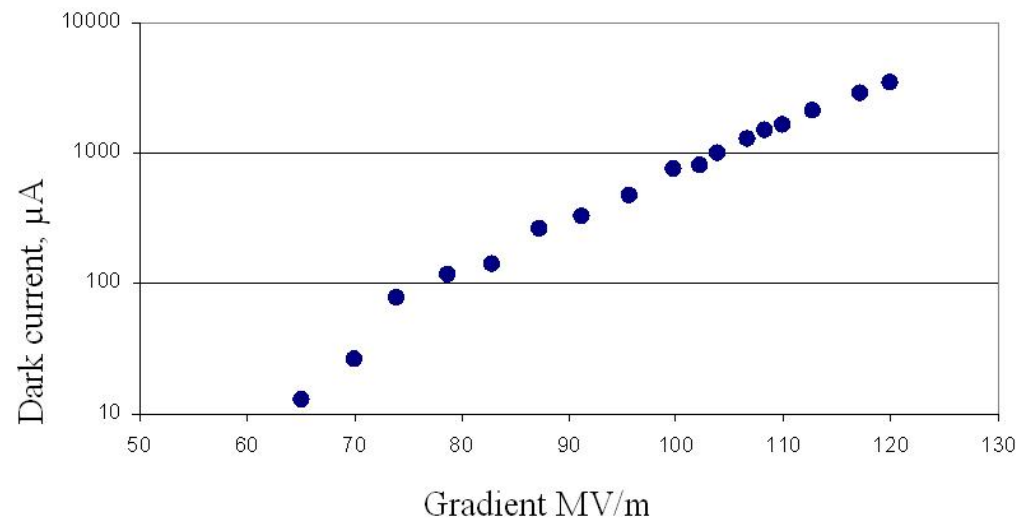

FIGURE 2. Dark current measurements of the gun.

The Fowler-Nordheim fit (Fig. 2) results in $\beta=84$, which agrees with one volt per angstrom figure of merit $\left(\beta \cdot E_{\max }=10.08 \mathrm{GV} / \mathrm{m}\right)$.

The beamline will contain various diagnostics. It includes RF monitoring, ion gauge, and a spectrometer for energy measurements. In the event of a breakdown, there will be a significant increase of reflected power, accompanied by a fast degradation of the vacuum level. We intend to capture and record this event in several ways. There will be a YAG screen for the measurement of the dark current distribution, a Faraday cup for an absolute charge measurement, and an RF pickup probe or a streak camera for time structure of the current and bunch length measurements. We have a gated ICCD camera which can be used to observe the breakdown process as it is triggered by IR or UV laser.

\section{SUMMARY}

We have developed a program for dark current and breakdown studies. A dedicated facility for this project is being built. It is an L-band electron gun with a diagnostics chamber. We intend to study the dark current emission and its relationship to the RF breakdown and physics of RF conditioning. In particular, we are going to take 'before' and 'after' the shots of the cathode to see if we can identify emitters and understand the nature of field enhancement.

This work is supported by the US Department of Energy under Contract No. W31-109-ENG-38 and National Science Foundation grant \# 0237162.

\section{REFERENCES}

1. Z. M. Yusof, M. E. Conde, and W. Gai, Phys. Rev. Lett. 93, 114801 (2004).

2. P. Wilson, SLAC-TN-06-003 (2006).

3. J. Norem, A. Hassanein, Z. Insepov, I. Konkashbaev, Proceedings of PAC2005, p. 1886 (2005).

4. R.H. Fowler and L. Nordheim, Proc. R. Soc. Lon. Ser-A 119, 173 (1928).

5. C.H. Ho, S.Y. Ho, G.Y. Hsiung et al., Proceedings of LINAC98, p. 520 (1999). 